\title{
Exploring cell membrane water exchange in aquaporin-4-deficient ischemic mouse brain using diffusion-weighted MRI
}

Takuya Urushihata' ${ }^{1}$, Hiroyuki Takuwa', Manami Takahashi', Jeff Kershaw², Yasuhiko Tachibana², Nobuhiro Nitta², Sayaka Shibata ${ }^{2}$, Masato Yasui ${ }^{3}$, Makoto Higuchi ${ }^{1}$ and Takayuki Obata ${ }^{2^{*}}$ (i)

\begin{abstract}
Background: Aquaporin-4 is a membrane channel protein that is highly expressed in brain astrocytes and facilitates the transport of water molecules. It has been suggested that suppression of aquaporin-4 function may be an effective treatment for reducing cellular edema after cerebral infarction. It is therefore important to develop clinically applicable measurement systems to evaluate and better understand the effects of aquaporin-4 suppression on the living body.

Methods: Animal models of focal cerebral ischemia were created by surgically occluding the middle cerebral artery of wild-type and aquaporin-4 knockout mice, after which multi-b-value multi-diffusion-time diffusion-weighted imaging measurements were performed. Data were analyzed with both the apparent diffusion coefficient (ADC) model and a compartmental water-exchange model.

Results: ADCs were estimated for five different $b$ value ranges. The ADC of aquaporin- 4 knockout mice in the contralateral region was significantly higher than that of wild-type mice for each range. In contrast, aquaporin-4 knockout mice had significantly lower ADC than wild-type mice in ischemic tissue for each b-value range.

Genotype-dependent differences in the ADC were particularly significant for the lowest ranges in normal tissue and for the highest ranges in ischemic tissue. The ADCs measured at different diffusion times were significantly different for both genotypes. Fitting of the water-exchange model to the ischemic region data found that the waterexchange time in aquaporin-4 knockout mice was approximately 2.5 times longer than that in wild-type mice.

Conclusions: Multi-b-value multi-diffusion-time diffusion-weighted imaging may be useful for in vivo research and clinical diagnosis of aquaporin-4-related diseases.
\end{abstract}

Keywords: Aquaporin 4, Cell membrane, Cerebral infarction, Diffusion magnetic resonance imaging, Experimental animal models

\footnotetext{
* Correspondence: obata.takayuki@qst.go.jp

${ }^{2}$ Applied MRI Research, Department of Molecular Imaging and Theranostics,

Institute for Quantum Medical Science, QST, Chiba 263-8555, Japan

Full list of author information is available at the end of the article
}

\section{Springer Open}

(c) The Author(s). 2021 Open Access This article is licensed under a Creative Commons Attribution 4.0 International License, which permits use, sharing, adaptation, distribution and reproduction in any medium or format, as long as you give appropriate credit to the original author(s) and the source, provide a link to the Creative Commons licence, and indicate if changes were made. The images or other third party material in this article are included in the article's Creative Commons licence, unless indicated otherwise in a credit line to the material. If material is not included in the article's Creative Commons licence and your intended use is not permitted by statutory regulation or exceeds the permitted use, you will need to obtain permission directly from the copyright holder. To view a copy of this licence, visit http://creativecommons.org/licenses/by/4.0/. 


\section{Key points}

- We investigated aquaporin-4 loss and focal brain ischemia with diffusion-weighted magnetic resonance imaging in a mouse animal model.

- Cell membrane water exchange time parameters were estimated by model fitting.

- The effect of aquaporin-4 loss is opposite in healthy and ischemic tissue.

- Diffusion-weighted magnetic resonance imaging may be useful for research and clinical diagnosis of aquaporin-4-related diseases.

\section{Background}

Aquaporin-4 is a membrane channel protein that allows water molecules to be passively transported, is the most commonly expressed aquaporin on the feet of astrocytes in the mammalian brain $[1,2]$, and plays an important function in water movement across the blood-brain barrier [3-6]. It has also been reported that aquaporin-4 is involved in brain diseases such as ischemic stroke $[7,8]$, hydrocephalus [9, 10], Alzheimer's disease [11-13], amyotrophic lateral sclerosis [14], traumatic brain injury [15], and epilepsy [16, 17]. These findings have led to the design of treatment strategies that target aquaporin$4[18,19]$.

Cytotoxic edema, a condition where increased water in brain cells causes swelling, is frequently observed in a variety of brain diseases $[7,20]$. Unfortunately, the molecular and cellular mechanisms underlying the formation and resolution of edema are not yet fully understood, and there is still no clear treatment [20, 21]. In studies using animal models, aquaporin-4 expression is sharply increased in ischemic brain edema [22, 23], and aquaporin-4 knockout or inhibitor administration has been shown to be effective in reducing cellular edema [8, 24-26]. Aquaporin-4 inhibition has therefore been proposed as a treatment for cytotoxic edema. On the other hand, inhibition of aquaporin- 4 is also known to cause astrocyte dysfunction, preventing recovery from ischemia [27-29]. The complex effects of aquaporin-4 on the formation of cytotoxic edema have not been fully evaluated, nor has a clear method for in vivo evaluation been established. A clinically applicable method that can reliably evaluate changes in cytotoxic edema caused by aquaporin-4 inhibition is needed for the future development of suitable drugs and other therapies.

Diffusion-weighted imaging (DWI) has been used as an important tool for the diagnosis of diseases such as stroke and cancer [30]. A quantity that is often estimated in DWI studies is the apparent diffusion coefficient (ADC). The ADC is said to be "apparent" because the complexity of in vivo tissue microstructure dictates that it is an indicator of the overall signal behavior from multiple diffusion-related processes, rather than the diffusion coefficient of a single water compartment. In fact, different ADCs may be attributed to different parts of an organism depending on the nature of diffusion in that component [31]. For example, as intracellular water diffusion is strongly obstructed by the cell membrane and many other structures inside the cell, it usually corresponds to a restricted diffusion component. On the other hand, diffusion through the extracellular space is comparatively, but not completely, free so it contributes to a hindered diffusion component. In addition, the disordered arrangement of the microvasculature implies that blood-water molecules display a diffusion-like behavior called intravoxel incoherent motion (IVIM). Accordingly, blood signal is attributed to an IVIM pseudo-diffusion component [32]. In general, the signals from each of these components contribute to the overall signal at low $b$ values. However, the contribution from the IVIM component is negligible for $b$ values above 500 $\mathrm{s} / \mathrm{mm}^{2}$, and the signal from the hindered diffusion component is relatively small for $b$ values greater than $2,000 \mathrm{~s} / \mathrm{mm}^{2}$, which leaves the restricted diffusion component near the cell as the dominant contributor at high $b$ values $[31,33]$. Therefore, observing the signal over a wide range of $b$ values may help to isolate the contributions of different diffusion components, in particular the restricted diffusion component, which is the component most likely to reflect the effects of aquaporin-4 on water transport.

In a previous study, we proposed and applied a DWIbased technique to quantitatively evaluate cell membrane water permeability for in vitro monoclonal cell suspensions [34]. The technique is based on a modification of the Andrasko-Kärger model [35-37], which is a simple two-compartment model with intercompartmental compound exchange. Briefly, since the effect of membrane permeability on DWI measurements is highly dependent on diffusion-time [38, 39], the water exchange-time between compartments was estimated from differences in DWI signal attenuation at different diffusion-times. It was shown that the technique can be used to characterize differences in water exchange between aquaporin-4-expressing and non-expressing cells, and the results were consistent with data measured by coherent anti-Stokes Raman scattering microscopy [40]. This noninvasive DWI-based method may also be useful for the evaluation of cell membrane water permeability changes caused by aquaporin- 4 abnormalities, and for the development of medicines targeting aquaporin-4.

In this study, we performed DWI on wild-type and aquaporin-4 knockout mice to evaluate the effects of aquaporin-4 suppression in vivo. Animal models with focal cerebral ischemia were surgically created by middle cerebral artery occlusion (MCAO), and multi- $b$-value 
multi-diffusion-time (MbMTd) DWI measurements were performed. ADCs were estimated and compared between the wild-type and aquaporin-4 knockout mice for both the ischemic and contralateral regions. The ischemic data was also analyzed with the twocompartment exchange model to quantitatively compare the water-exchange time and the other model parameters between mice with and without aquaporin- 4 .

\section{Methods}

All experiments were performed in accordance with the institutional guidelines on humane care and use of laboratory animals and were approved by the Institutional Committee for Animal Experimentation of the National Institutes for Quantum and Radiological Science and Technology (QST). The datasets analyzed during the current study are available from the corresponding author on reasonable request.

\section{Animal preparation}

A total of 6 C57BL/6J wild-type mice (both male and female, 20-30 g, 8-10 weeks; Japan SLC, Hamamatsu, Japan), and 7 aquaporin-4 knockout mice (both male and female, 20-30 g, 8-10 weeks) generated as described previously [41, 42] (acc. no. CDB0758 K: http:// www.cdb.riken.jp/arg/mutant\%20mice\%20list.html), were used in the magnetic resonance imaging (MRI) experiments. All mice were housed individually in separate cages with water and food ad libitum. Mouse cages were kept at a temperature of $25^{\circ} \mathrm{C}$ in a 12-h light/dark cycle. Overall, no clear differences in body weight and size were observed for any of the mice. MCAO was performed for all animals using the Tamura method [43], where a permanent occlusion is made at the proximal branch of the MCA in the left cerebral cortex. In this animal model, ischemia in the MCA region occurs soon after MCAO, and the infarction expands and peaks at 24 hours after surgery [43-46].

\section{MRI measurements}

MRI measurements were performed at $2 \mathrm{~h}$ after MCAO surgery, which is during the initial stage of cytotoxic edema formation due to ischemia. This time window was selected because it was anticipated that the presence or absence of aquaporin-4 would contribute to differences in ischemic-related water movement during edema formation and before blood-brain barrier breakdown [36 , 47]. All measurements were performed with a 7-T animal MRI (Kobelco and Bruker, Tokyo, Japan). The mice were initially anesthetized with $3.0 \%$ isoflurane (Escain, Mylan Japan, Tokyo, Japan), and then with 1.5\% to $2.0 \%$ isoflurane and a $1: 5$ oxygen/room-air mixture during the MRI experiments. Rectal temperature was continuously monitored with an optical fiber thermometer (FOT-M, FISO, Quebec, QC, Canada), and maintained at $37.0 \pm 0.5{ }^{\circ} \mathrm{C}$ (range) using a heating pad (Temperature control unit, Rapid Biomedical, Rimpar, Germany). Warm air was provided with a homemade automatic heating system regulated by an electric temperature controller (E5CN, Omron, Kyoto, Japan) throughout all experiments. During MRI scanning, the mice lay in a prone position on a MRI-safe cradle and were held in place with handmade ear bars.

MbMTd DWI was obtained using a pulsed-gradient spin-echo sequence with four-shot echo-planar acquisition (repetition time $3 \mathrm{~s}$, echo time $115 \mathrm{~ms}$, matrix size $128 \times 128$, spatial resolution $0.02 \times 0.02 \mathrm{~mm}^{2}$, slice thickness $1.5 \mathrm{~mm}$, gradient directions 3 ). The separation of the diffusion-gradient lobes $(\Delta)$ was set at 40,70 , and $100 \mathrm{~ms}$ to vary diffusion-time while keeping echo time constant. The diffusion-gradient duration $(\delta)$ was fixed at $7 \mathrm{~ms}$ for all experiments. For the pulsed-gradient spin-echo sequence, $\Delta-\delta / 3$ is usually taken to represent the diffusion-time. For each $\Delta$, the $b$ value was increased from 0 to $8,000 \mathrm{~s} / \mathrm{mm}^{2}$ in 11 steps $(0,2,500,1,000$, 2,000, 3,000, 4,000, 5,000, 6,000, 7,000, and 8,000 s/mm ${ }^{2}$ ) by increasing the gradient amplitude. The multi- $b$-value DWI scan time for each $\Delta$ was about 6 min, which means that it took about $18 \mathrm{~min}$ for one set of MbMTd DWI. To check scan stability, 4 sets of MbMTd DWI were acquired for each animal in the study.

\section{DWI data processing}

DWI data analysis was performed in MATLAB, version R2019a (MathWorks, Natick, MA, USA). Regions-ofinterest (ROIs) were drawn in the ischemic and contralateral regions on T2-weighted images. The DWI data were averaged over the three gradient directions, and ADC maps were created by fitting to the logarithmic signals with respect to b-value using ordinary least squares. ADCs were estimated for five different $b$ value ranges: $0-2,000 \mathrm{~s} / \mathrm{mm}^{2}$, which is used in general clinical practice; $500-2,000 \mathrm{~s} / \mathrm{mm}^{2}$, where it is thought the effect of the IVIM component is suppressed; and 2,000-4,000, $4,000-6,000$, and $6,000-8,000 \mathrm{~s} / \mathrm{mm}^{2}$, which are ranges where the restricted diffusion component is expected to be dominant. The average value of the ADC was calculated for each ROI and then averaged over animals. The signal-to-noise ratio (SNR) of the data was estimated using the air signal method [48],

$$
\mathrm{SNR}=\left(\frac{\pi}{2}\right)^{\frac{1}{2}} \times \frac{S_{p}}{S_{\text {air }}},
$$

where $S_{\mathrm{p}}$ is the signal in the ROI and $S_{\text {air }}$ is the signal from a background region.

Evaluation of cell membrane water exchange-time was performed using a two-compartmental model with inter- 
compartment exchange (Fig. S1) [34]. Briefly, the model assumes that $\Delta$ is sufficiently long that the diffusion coefficient in the extracellular space $\left(D_{\text {ex }}\right)$ is approximately constant, while the diffusion coefficient in the intracellular space $\left(D_{\text {in }}\right)$ is inversely proportional to the diffusion time. The data was then analyzed using a constant $D_{\mathrm{ex}}$, and $D_{\text {in }}$ was dependent on diffusion-time as below,

$$
D_{i n}=\frac{\alpha}{(\Delta-\delta / 3)^{\beta}} .
$$

In this equation, $\alpha$ has dimensions of length squared in the case that $\beta=1$, and $\beta$ is a parameter inserted to test the assumption that $D_{\text {in }}$ is inversely proportional to $\Delta-\delta /$ 3. Using the modified model, the DWI-based estimate of the exchange-time $\left(\tau_{M R I}\right)$ is,

$$
\tau_{\mathrm{MRI}}=F_{e x} \cdot t_{\mathrm{in}}=F_{\mathrm{in}} \times t_{e x},
$$

where $F_{\mathrm{ex}}$ and $F_{\text {in }}$ are the signal fractions of the extracellular and intracellular spaces, respectively, and $t_{\mathrm{ex}}$ and $t_{\mathrm{in}}$ are the lifetimes in the extracellular and intracellular spaces, respectively. Since it was demonstrated that $\beta$ is close to 1 in a previous study [34], $\beta$ was set to 1 in this study. The parameters $t_{\mathrm{in}}, F_{\mathrm{in}}, D_{\mathrm{ex}}$, and $\alpha$ were used as the free parameters while fitting to the model. $F_{\mathrm{ex}}, t_{\mathrm{ex}}$, and $\tau_{M R I}$ were then obtained using Eq. 3 and the constraint $F_{\text {ex }}+F_{\text {in }}=1$.

\section{Statistical analysis}

Statistical analyses were performed with the Statistics and Machine Learning Toolbox of MATLAB, version R2019a (MathWorks, Natick, MA, USA). Normalized DWI signals are presented as mean \pm standard deviation over animals (Fig. 1). The ADCs and exchange model parameters are plotted for each animal with a bar indicating the mean over animals (Figs. 2, 3, and 4). Twoway analysis of variance (ANOVA), with mouse genotype and $\Delta$ as independent variables, was performed for the ADC estimated for each $b$ value range. In addition, Student's $t$ test was used to compare the exchangemodel parameters between mouse genotype. The normality of each data set was confirmed with a Kolmogorov-Smirnov test. A $p$ value $<0.05$ was interpreted as being significant.

\section{Results}

\section{MbMTd DWI signal changes caused by aquaporin- 4 suppression and ischemia}

As demonstrated by the high area on T2-weighted and diffusion-weighted images, MCAO produced a focal ischemic region on the left side of the mouse brain for both the wild-type and aquaporin-4 knockout mice (Fig. 1a). ROI-based analysis was performed on the ischemic (Fig. 1b) and the contralateral (Fig. 1c) regions of the
MbMTd DWI, and the normalized signal was plotted against $b$ value for all three values of $\Delta(40,70$, and 100 $\mathrm{ms})$. In the ischemic region, the aquaporin-4 knockout mice had less $b$ value dependent signal attenuation than the wild-type mice for all $\Delta \mathrm{s}$ (Fig. 1b). On the other hand, in the contralateral region, the aquaporin- 4 knockout mice had greater $b$ value dependent signal attenuation than the wild-type mice for all $\Delta s$ (Fig. 1c). Longer $\Delta$ corresponded to greater signal attenuation for both mouse types and both regions (Fig. S2). The SNR in the contralateral region was less than 5 for $b$ values above $5,000 \mathrm{~s} / \mathrm{mm}^{2}$, so that data was excluded from subsequent analysis of that region (Fig. S3).

\section{Apparent diffusion coefficient}

To characterize diffusion in the ischemic regions of both mouse types, ADCs were calculated for five $b$ value patterns. Representative animal ADC maps are shown in Fig. 2a. For all $b$ value ranges, the ADCs were significantly lower for the aquaporin-4 knockout mice than for the wild-type mice (Table 1). Moreover, the ADCs for the $2,000-4,000,4,000-6,000$, and $6,000-8,000 \mathrm{~s} / \mathrm{mm}^{2} b$ value ranges showed a significant $\Delta$-dependent difference (Fig. 2b, Table 1). The ADC in the ischemic region showed no significant interaction between mouse genotype and $\Delta$ for all $b$ value ranges.

As the SNR was very low above $b=5,000 \mathrm{~s} / \mathrm{mm}^{2}$ (Fig. $\mathrm{S} 3)$, the ADC in the contralateral region was calculated for only three of the $b$ value ranges $(0-2,000,500-2,000$, and 2,000-4,000 s/ $\mathrm{mm}^{2}$ ). Representative ADC maps are presented in Fig. 3a. In contrast to the ischemic region, the aquaporin-4 knockout mice showed significantly higher ADC than the wild-type mice for each of the $b$ value patterns (Fig. 3b, Table 2). Only the ADC for the $2,000-4,000 \mathrm{~s} / \mathrm{mm}^{2} b$ value range showed a $\Delta$-dependent difference. The ADC for all three $b$ value ranges showed no significant interaction between mouse genotype and $\Delta$ (Fig. 3b, Table 2).

\section{Parameters of the compartmental exchange model}

Estimates of the four parameters ( $\tau_{\mathrm{MRI}}, \alpha, F_{\mathrm{in}}$, and $D_{\mathrm{ex}}$ ) obtained by fitting the exchange model to the ischemic data are shown in Fig. 4. The aquaporin-4 knockout mice showed a significantly longer water exchange-time than the wild-type mice $(p<0.006)$. The other parameters, $\alpha, F_{\mathrm{in}}$, and $D_{\mathrm{ex}}$, had larger values for the wild-type mice (Fig. $4 \mathrm{~b}-\mathrm{d})$, with the difference being significant $(\alpha$, $\left.p<0.003 ; F_{\mathrm{in}}, p<0.006 ; D_{\mathrm{ex}}, p<0.044\right)$.

\section{Discussion}

In this study, we measured MbMTd DWI and compared ADCs estimated for ischemic and normal tissue of aquaporin-4 knockout and wild-type mice. In the ischemic region, aquaporin-4 knockout mice showed lower 


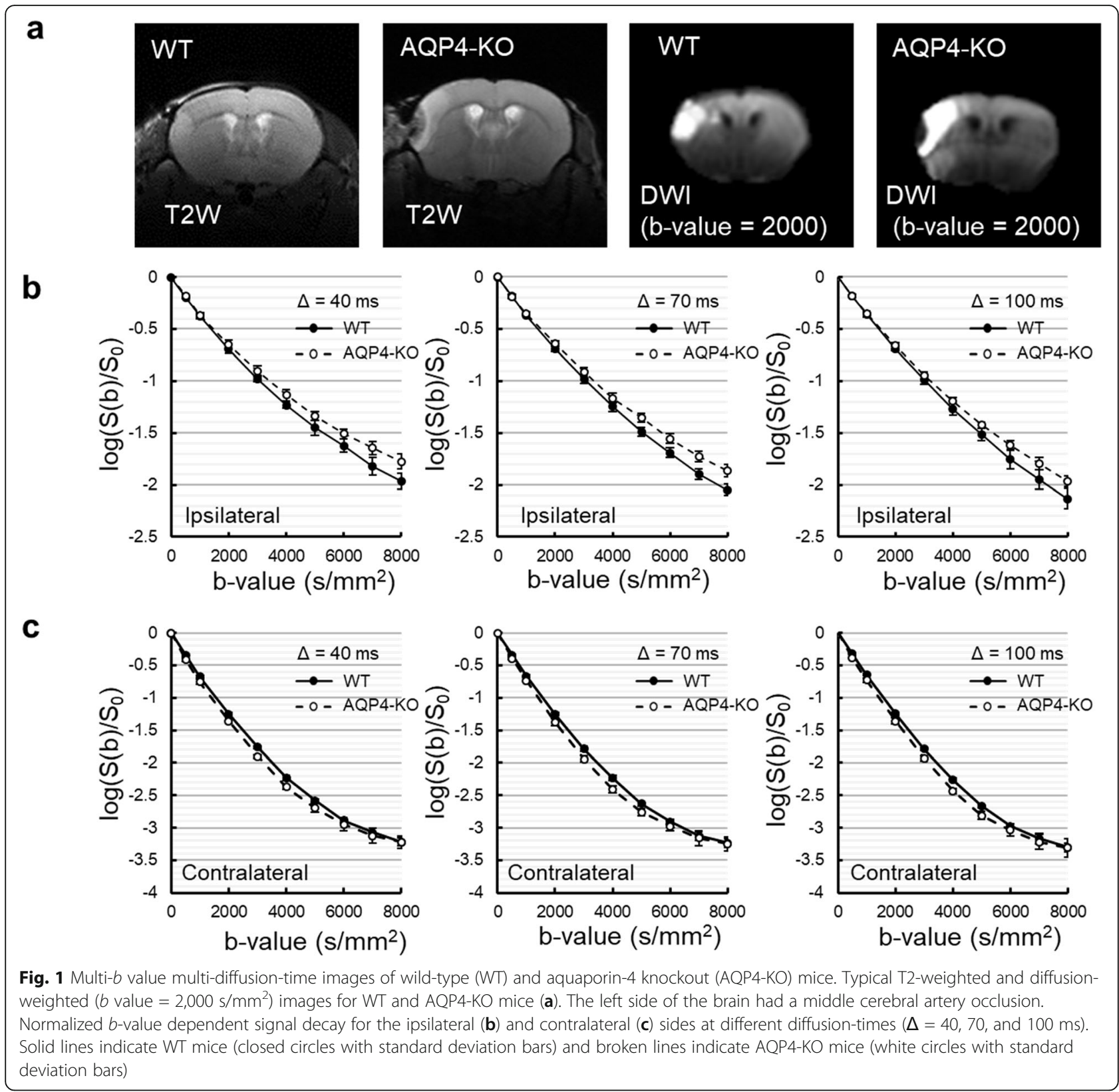

ADCs compared to wild-type mice, whereas higher ADCs were observed in the contralateral region. In addition, we compared parameter estimates obtained by fitting a two-compartment exchange model to the DWI signal. The cell membrane water exchange-time was approximately 2.5 times longer for the aquaporin- 4 knockout mice than for the wild-type mice.

The observation of larger ADCs in normal tissue for the aquaporin-4 knockout mice is consistent with a report of an increase in ADC after the administration of the aquaporin-4 inhibitor TGN-020 [49]. It has also been reported that the volume of the extracellular space is comparatively large in aquaporin-4 knockout mice [50], and the suppression of aquaporin-4 expression by siRNAs in cultured rat cells reduces the size and total number of astrocytes [51]. As the contribution of the hindered diffusion component is relatively small for $b$ values over $2,000 \mathrm{~s} / \mathrm{mm}^{2}$, our observation of a larger difference between the contralateral ADCs of the wild-type and aquaporin- 4 knockout mice for the two sub-2,000 s/ $\mathrm{mm}^{2} b$ value ranges than for the $2,000-4,000 \mathrm{~s} / \mathrm{mm}^{2} b$ value range (Fig. 3b, Table 2) may therefore reflect differences in the extracellular volume fraction between wild-type and aquaporin-4 knockout mice. Furthermore, 
a

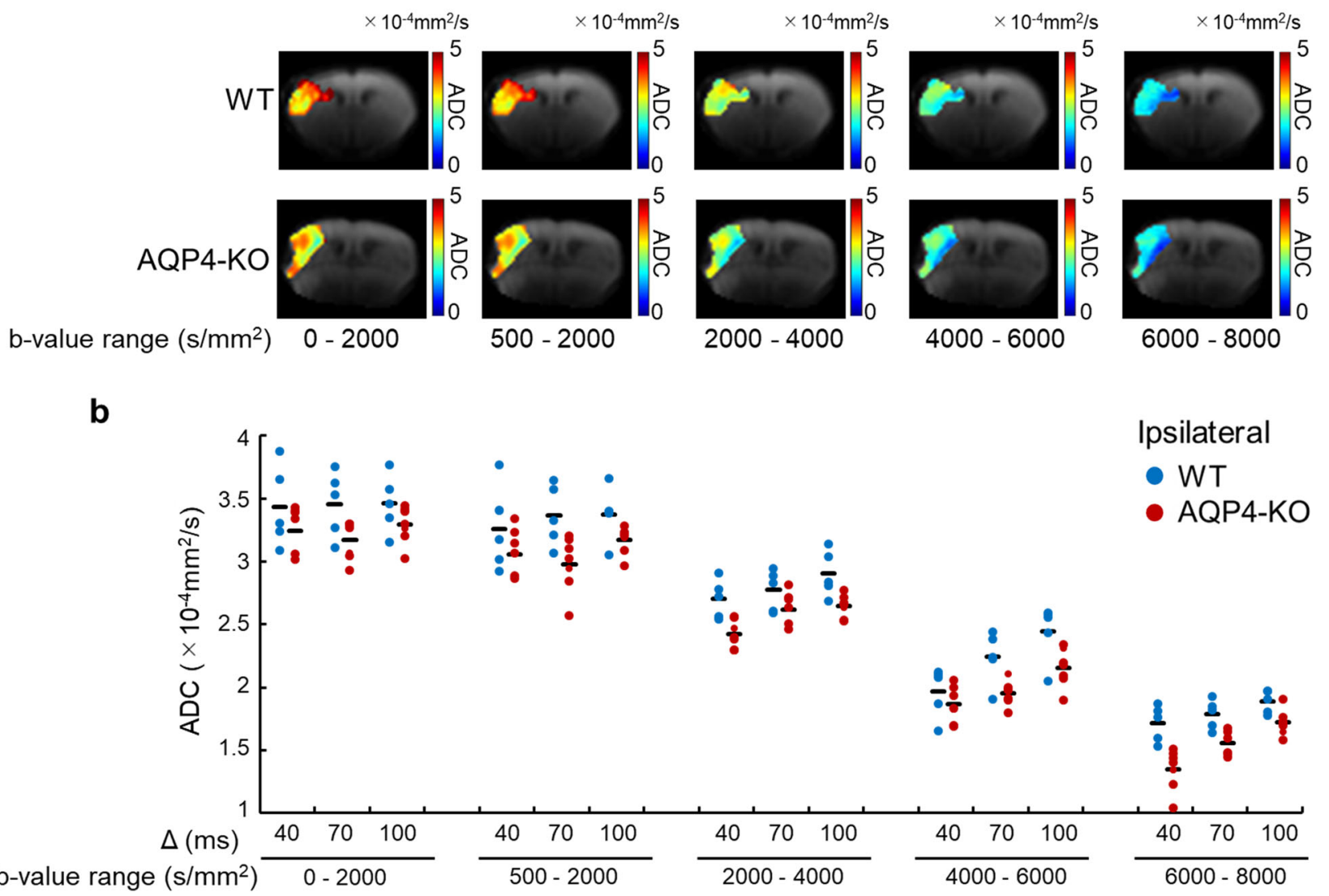

Fig. 2 Apparent diffusion coefficient (ADC) in the ischemic region. Typical ADC maps of wild-type (WT) (top) and aquaporin-4 knockout (AQP4$\mathrm{KO}$ ) mice (top and bottom, respectively) focused on the ipsilateral region at $\Delta=70 \mathrm{~ms}(\mathbf{a})$. The ADC in the ischemic region has a range of $0-5 \times$ $10^{-4} \mathrm{~mm}^{2} / \mathrm{s}$. The plots show the mean ADC of each WT (blue) and AQP4-KO (red) mouse for each $b$-value range and $\Delta$ (b). The black bars correspond to the mean over animals. The results of the statistical analysis are shown in Table 1

as aquaporin-4 is expressed normally in wild-type mice, it might be hypothesized that the ADC of the restricted diffusion component in normal tissue is larger than it is for APQ4-KO mice. Unfortunately, we were unable to confirm this due to the low SNR of the 4,000-6,000 and $6,000-8,000 \mathrm{~s} / \mathrm{mm}^{2}$ data on the contralateral side, but the trend of a decreasing difference between the ADCs of the two mouse genotypes with increasing $b$ value range is consistent with the hypothesis (Fig. 3b, Table 2).

In ischemic tissue, the ADCs of the aquaporin-4 knockout mice were smaller than those of the wild-type animals for all $b$ value ranges (Fig. 2b). Moreover, the gap between the ADCs of the two genotypes tended to become more significant for the higher $b$ value ranges (Table 1). Ischemia-induced swelling of astrocytes and neuronal dendrites is expected to increase the volume fraction of the restricted diffusion component regardless of genotype [52, 53], in which case it is likely that the contribution of that component to the overall signal has greater weighting across all $b$ value ranges. Therefore, it is possible that our observations in ischemic tissue reflect differences in the ADC and cell membrane water exchange-time of the restricted diffusion compartment for the two mouse types.

As the DWI signal can be affected by cerebral blood flow when using low $b$ values $[32,54,55]$, we compared the ADCs estimated using the $0-2,000$ and $500-2,000 \mathrm{~s} /$ $\mathrm{mm}^{2} b$ value ranges. The ADCs were similar for the two ranges, indicating that differences dependent on mouse type were not related to the effect of cerebral blood flow on DWI.

The exchange model parameters in the living mouse brain were estimated by fitting to the MbMTd DWI data in the ischemia region (Fig. 4). Exchange time was about 2.5 times and significantly longer for the aquaporin- 4 knockout mice than for the wild-type mice (Fig. 4a), which is consistent with previous in vitro studies [34, 40]. In addition, both the intracellular fraction, $F_{i n}$, and the extracellular diffusion coefficient, $D_{\mathrm{ex}}$, estimates for the wild-type mice were greater than those for the 


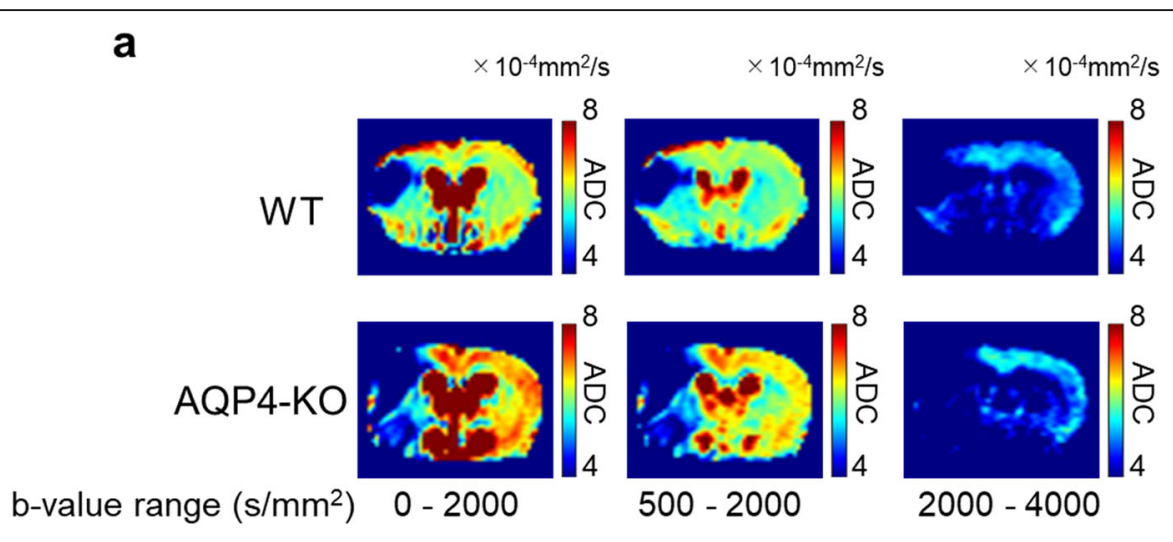

b

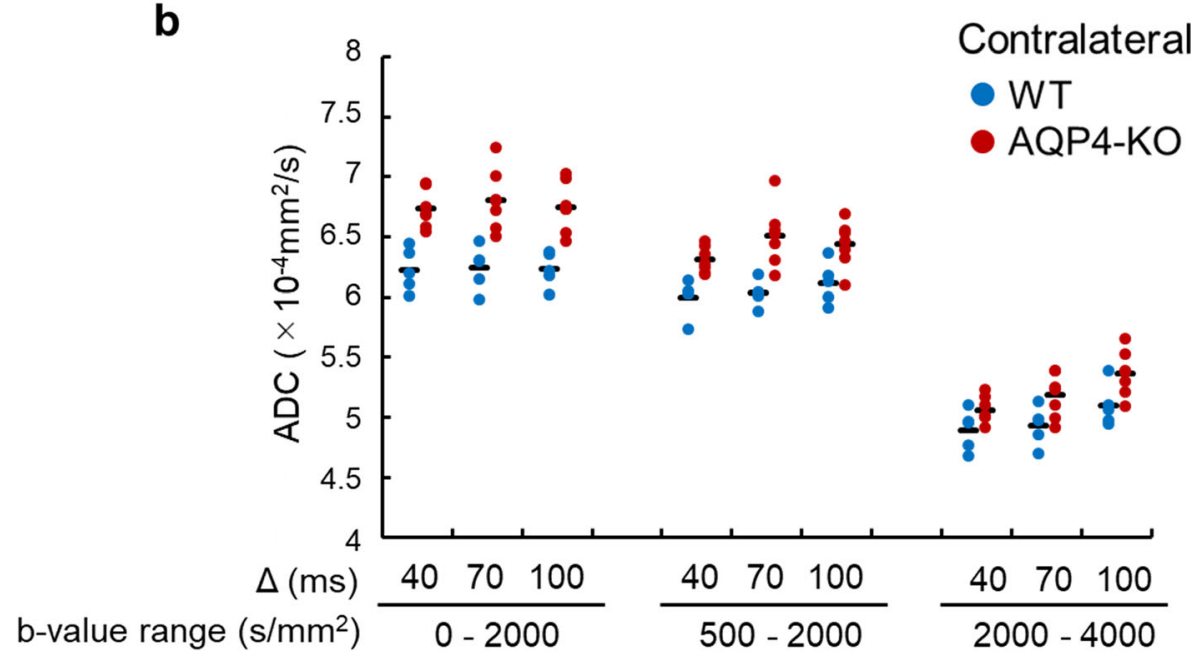

Fig. 3 Apparent diffusion coefficient (ADC) in the contralateral region. Typical ADC maps of wild-type (WT) and aquaporin-4 knockout (AQP4-KO) mice (top and bottom, respectively), focused on the contralateral region (a). The ADC in the contralateral region has a range of $4-8 \times 10^{-4} \mathrm{~mm}^{2} / \mathrm{s}$. The plots show the mean ADC of each WT (blue) and AQP4-KO (red) mouse for each $b$-value range and $\Delta(\mathbf{b})$. The black bars correspond to the mean over animals. The results of the statistical analysis are shown in Table 2 . The ADC maps and bar graphs for $b=4,000-6,000$, and $6,000-8,000 \mathrm{~s} / \mathrm{mm}^{2}$ are excluded because of low signal-to-noise ratio (see Fig. S3)

APQ4-KO mice (Fig. 4c, d). This result for $D_{\text {ex }}$ is a little unexpected as it could be argued that the smaller extracellular fraction, $F_{\mathrm{ex}}=1-F_{\mathrm{in}}$, of the wild-type mice would result in a smaller value for the extracellular diffusion coefficient [56]. However, it is also possible that $D_{\text {ex }}$ is larger for wild-type mice due to the need to break hydrogen bonds in clusters of water molecules so that a single molecule can pass through an aquaporin-4 channel [57-59]. Our results suggest that cell membrane water exchange abnormalities caused by aquaporin-4 loss might be detected in living animals.

A previous in vitro study found that signal attenuation for aquaporin- 4 non-expressed cells at high $b$ values $(\approx$ $4,000-8,000 \mathrm{~s} / \mathrm{mm}^{2}$ ) consistently decreased with increasing diffusion-time [34], which is similar to the behavior expected for a system where restricted diffusion dominates [60]. In the same in vitro study, attenuation of the DWI signal for aquaporin-4-expressed cells did not show a consistent trend with respect to increasing diffusiontime, from which it was suggested that the permeability of the cell membrane may have a significant effect on the signal at high $b$ values. In contrast to those in vitro results, it was observed for the in vivo experiments performed here that the signal attenuation in ischemic tissue at high $b$ values increased with increasing diffusion-time for both genotypes (Figs. 1 and S2). A similar observation was made in human subjects with stroke lesions [38].

The in vivo measurements of water exchange-time made for this work are shorter than those made on cultured cells using the same technique [34]. The reason for this result is not yet clear, but several factors may be involved. First, it may simply be that the water exchange-time of in vivo mouse cells is shorter than that of cultured cells regardless of aquaporin-4 expression. Also, it has been reported that acid $\mathrm{pH}$ increases the 

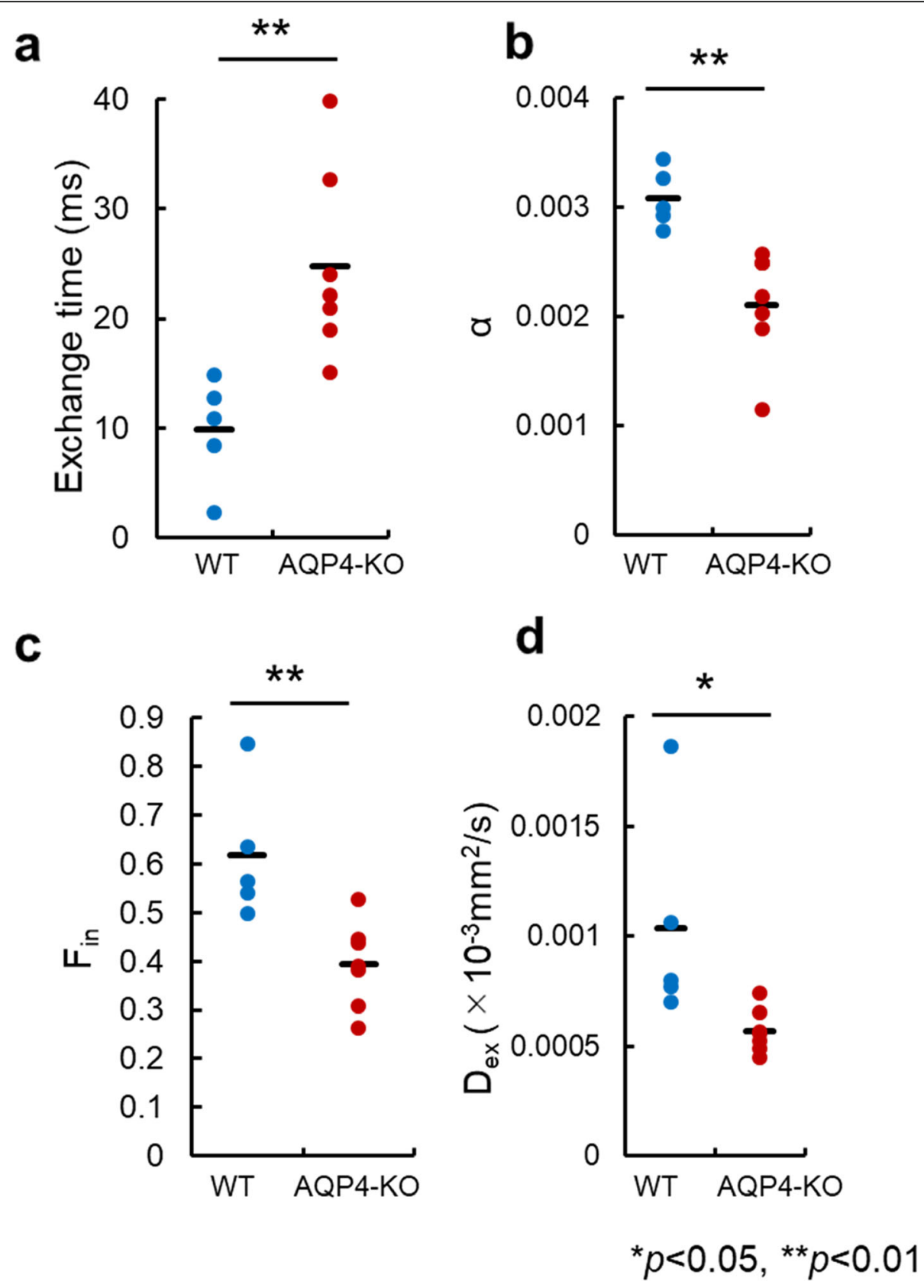

Fig. 4 Estimates of the exchange model parameters in the ischemic region. The exchange model parameters were estimated by fitting to the diffusion-weighted imaging data in the ischemic region. Exchange-time (a), water exchange time between the intracellular and extracellular space; $a(\mathbf{b})$, a parameter reflecting the mean cell volume; $F_{\text {in }}(\mathbf{c})$, signal fraction from intracellular space. $D_{\text {ex }}(\mathbf{d})$, apparent diffusion coefficient for the extracellular space. The plots show the mean for each wild-type (WT) (blue) and aquaporin-4 knockout (AQP4-KO) (red) mouse, while the black bars correspond to the mean over animals. ${ }^{*}$ and ${ }^{* *}$ indicate significant differences in the estimates from the WT and AQP4-KO mice $(\mathbf{a}, p=0.006 ; \mathbf{b}, p=0.003 ; \mathbf{c}, p=0.004 ; \mathbf{d}, p=0.044 ;$ unpaired $t$ test $)$

Table 1 Two-way ANOVA results ( $F$ values and $p$ values) for the ADC on the ipsilateral side

\begin{tabular}{|c|c|c|c|c|c|}
\hline$b$ value $\left(\mathrm{s} / \mathrm{mm}^{2}\right)$ & $0-2,000$ & $500-2,000$ & $2,000-4,000$ & $4,000-6,000$ & $6,000-8,000$ \\
\hline Genotype & $8.66(0.006)^{a}$ & $12.4(0.001)^{a}$ & $24.9(<0.001)^{\mathrm{a}}$ & $15.4(<0.001)^{\mathrm{a}}$ & $37.9(<0.001)^{\mathrm{a}}$ \\
\hline$\Delta$ & $0.244(0.785)$ & $0.938(0.402)$ & $9.92(0.003)^{a}$ & $15.1(<0.001)^{\mathrm{a}}$ & $14.7(<0.001)^{\mathrm{a}}$ \\
\hline Genotype $\times \Delta$ & $0.241(0.787)$ & $0.675(0.531)$ & $0.684(0.512)$ & $1.17(0.323)$ & $2.08(0.142)$ \\
\hline
\end{tabular}

The numbers within parentheses are $p$ values, and those less than 0.05 were interpreted as being statistically significant $\left({ }^{\mathrm{a}}\right)$. $A D C$ Apparent diffusion coefficient, ANOVA Analysis of variance 
Table 2 Two-way ANOVA results ( $F$ values and $p$ values) for the ADC on the contralateral side

\begin{tabular}{llll}
\hline $\boldsymbol{b}$ value $\left(\mathbf{s} / \mathbf{m m}^{\mathbf{2}}\right)$ & $\mathbf{0 - 2 , 0 0 0}$ & $\mathbf{5 0 0 - 2 , 0 0 0}$ & $\mathbf{2 , 0 0 0 - 4 , 0 0 0}$ \\
\hline Genotype & $64.5(<0.001)^{\mathrm{a}}$ & $38.85(<0.001)^{\mathrm{a}}$ & $16.4(<0.001)^{\mathrm{a}}$ \\
$\Delta$ & $0.171(0.844)$ & $1.896(0.168)$ & $6.86(0.004)^{\mathrm{a}}$ \\
Genotype $\times \boldsymbol{\Delta}$ & $0.0849(0.919)$ & $0.811(0.454)$ & $0.309(0.737)$
\end{tabular}

The numbers within parentheses are $p$ values, and those less than 0.05 were interpreted as being statistically significant $\left({ }^{\mathrm{a}}\right)$. $A D C$ Apparent diffusion coefficient, ANOVA Analysis of variance

water permeability of aquaporin [61, 62]. It is therefore possible that the higher acidity of ischemic tissue increased the water permeability of aquaporin and hence shortened the water exchange-time $[63,64]$. Finally, water exchange times have been reported to be shorter at higher temperatures [40], and the difference of the water exchange time between in vitro and in vivo experiments may reflect the differences in mouse body temperature $\left(36^{\circ} \mathrm{C}\right)$ and room temperature $\left(23^{\circ} \mathrm{C}\right)$.

Our study has limitations. The relatively long echo time required to perform long $\Delta$ scans results in low SNR at high $b$ values. Therefore, our method is limited to conditions where the DWI signal is quite high, such as in cerebral infarction, cancer, and white matter lesions. It is difficult to evaluate water permeability in the normal brain or in diseases such as Alzheimer's that are not associated with a high DWI signal. Other methods that utilize the kurtosis may be more useful for low SNR situations $[65,66]$. In addition, the limitations previously reported for the exchange model also apply here [34]. That is, the model does not consider the possibility that $\mathrm{T} 1$ and $\mathrm{T} 2$ are different in intracellular and extracellular spaces, nor does it consider the anisotropy of water diffusion. Finally, observations were made at only one point $2 \mathrm{~h}$ after MCAO. Even though there was no substantial change in signal between the four sets of MbMtd DWI measurements taken for each mouse (a period of about $1.5 \mathrm{~h}$, data not shown), the ischemic cascade is a dynamic process so a future longitudinal study of membrane permeability changes in MCAO model mice is important.

In conclusion, the present in vivo MbMtd DWI study found significant differences in the ADCs estimated across different $b$ value ranges for animal models with and without aquaporin-4 expression in normal and ischemic tissue. Genotype-dependent differences in the ADC were particularly significant for the low $b$ value range in normal tissue and for the high $b$ value ranges in ischemic tissue. A significant difference in the ADCs measured at different diffusion-times was detected for both genotypes. Permeability may make a major contribution to the diffusion time dependence of the ADC, but the dependence is independent of genotype. Estimation of the parameters of a water exchange model quantified differences in water exchange-time that might be related to aquaporin-4 deficiency. The results suggest that MbMtd DWI might be useful for evaluating the efficacy of aquaporin-4 knockout targeted medicines and for the clinical diagnosis of aquaporin-4-related diseases.

\section{Abbreviations}

ADC: Apparent diffusion coefficient; ANOVA: Analysis of variance;

DWI: Diffusion-weighted imaging; IVIM: Intravoxel incoherent motion; MbMTd: Multi-b-value multi-diffusion-time; MCAO: Middle cerebral artery occlusion; MRI: Magnetic resonance imaging; ROI: Regions-of-interest; SNR: Signal-to-noise ratio

\section{Supplementary Information}

The online version contains supplementary material available at https://doi. org/10.1186/s41747-021-00244-y.

Additional file 1: Supplementary figure. 1 The two-compartment model with inter-compartmental exchange. $C_{e x}(T d)$ and $C_{i n}(T d)$ are the normalized extracellular and intracellular signals, respectively, at diffusiontime $T d . t_{\text {ex }}$ and $t_{\text {in }}$ are constants representing the inter-compartmental lifetimes. $D_{\text {ex }}$ is the diffusion coefficient in the extracellular space. The diffusion coefficient in the intracellular space is modelled as " $\mathrm{a} /(\Delta-\delta / 3)^{\beta "}$, where $\beta$ is taken to be 1 in this work, and " $a$ " is a fitting parameter with units of length squared if $\beta=1$. The water signal from each compartment decreases with a rate constant equal to $q^{2} D$, where the $q$-value is determined by the parameters of the motion probing gradient. Supplementary figure. $\mathbf{2}$ The mean b-value dependent signal attenuation on each side of the brain. The data is the same as that shown in Figure 1 but plotted to highlight differences due to altered $\Delta$. (a) Ipsilateral side of wildtype (WT) mice, (b) ipsilateral side of aquaporin-4 knockout (AQP4-KO) mice, (c) contralateral side of WT and (d) contralateral side of AQP4-KO mice. Supplementary figure. $\mathbf{3}$ The b-value-dependent SNR on the ipsilateral (left) and contralateral (right) sides of the brain. The horizontal broken lines indicate $S N R=5$. Data were excluded from the analysis if the SNR was less than 5. SNR: signal-to-noise ratio

\section{Acknowledgements \\ The authors thank Takeharu Minamihisamatsu for help with animal management.}

\section{Authors' contributions}

TU and TO planned and conducted the study. HT and TM created an animal model. TU, NN, and SS performed MRI acquisition. TU and YT analyzed the data. TU and TO performed literature research and wrote the first draft of the manuscript. JK, MY, and MH supervised the final manuscript. All authors were involved in editing the manuscript and approved the final version.

\section{Funding}

This work was supported by a Grant-in-Aid for Scientific Research (KAKENHI, \#15H04910 [TO]) from the Japan Society for the Promotion of Science (JSPS).

\section{Availability of data and materials}

The datasets used and/or analyzed during the current study are available from the corresponding author on reasonable request.

\section{Declarations}

Ethics approval and consent to participate

All experiments were performed in accordance with the institutional guidelines on humane care and use of laboratory animals, and were approved by the Institutional Committee for Animal Experimentation of the National Institutes for Quantum and Radiological Science and Technology (QST).

Consent for publication

Not applicable. 


\section{Competing interests}

The authors declare that they have no competing interests.

\begin{abstract}
Author details
${ }^{1}$ Department of Functional Brain Imaging Research, Institute for Quantum Medical Science, QST, Chiba 263-8555, Japan. ${ }^{2}$ Applied MRI Research, Department of Molecular Imaging and Theranostics, Institute for Quantum Medical Science, QST, Chiba 263-8555, Japan. ${ }^{3}$ Keio Advanced Research Center for Water Biology and Medicine, Keio University, Tokyo 160-0016, Japan.
\end{abstract}

\section{Received: 14 June 2021 Accepted: 27 August 2021}

Published online: 07 October 2021

\section{References}

1. Badaut J, Brunet J-F, Regli $L$ (2007) Aquaporins in the brain: from aqueduct to "multi-duct." Metab Brain Dis 22:251-263. https://doi.org/10.1007/s11011007-9057-2, 3-4

2. Rash JE, Yasumura T, Hudson CS, Agre P, Nielsen S (1998) Direct immunogold labeling of aquaporin-4 in square arrays of astrocyte and ependymocyte plasma membranes in rat brain and spinal cord. Proc Natl Acad Sci U S A 95:11981-11986. https://doi.org/10.1073/pnas.95.20.11981, 20

3. Vajda Z, Pedersen M, Doczi T, Sulyok E, Nielsen S (2004) Studies of mdx mice. Neuroscience 129:991-996. https://doi.org/10.1016/J.NEUROSCIENCE.2 004.08.055, 4

4. Zhang Y, Xu K, Liu Y, et al (2019) Increased cerebral vascularization and decreased water exchange across the blood-brain barrier in aquaporin-4 knockout mice. PLoS One 14:. https://doi.org/10.1371/journal.pone.0218415, 6, e0218415

5. Pavlin T, Nagelhus EA, Brekken C, et al (2017) Loss or mislocalization of aquaporin-4 affects diffusion properties and intermediary metabolism in gray matter of mice. Neurochem Res 42:77-91. https://doi.org/10.1007/s11 064-016-2139-y, 1

6. Ohene Y, Harrison IF, Nahavandi P, et al (2019) Non-invasive MRI of brain clearance pathways using multiple echo time arterial spin labelling: an aquaporin-4 study. Neuroimage 188:515-523. https://doi.org/10.1016/j. neuroimage.2018.12.026

7. Aoki K, Uchihara T, Tsuchiya K, Nakamura A, Ikeda K, Wakayama Y (2003) Enhanced expression of aquaporin 4 in human brain with infarction. Acta Neuropathol 106:121-124. https://doi.org/10.1007/s00401-003-0709-y, 2

8. Manley GT, Fujimura M, Ma T, et al (2000) Aquaporin-4 deletion in mice reduces brain edema after acute water intoxication and ischemic stroke. Nat Med 6:159-163. https://doi.org/10.1038/72256, 2

9. Castañeyra-Ruiz L, González-Marrero I, González-Toledo JM, et al (2013) Aquaporin-4 expression in the cerebrospinal fluid in congenital human hydrocephalus. Fluids Barriers CNS 10. https:/doi.org/10.1186/2045-8118-10-18, 1

10. Mao X, Enno TL, Del Bigio MR (2006) Aquaporin 4 changes in rat brain with severe hydrocephalus. Eur J Neurosci 23:2929-2936. https://doi.org/10.1111/ j.1460-9568.2006.04829.x, 11

11. Xu Z, Xiao N, Chen Y, et al (2015) Deletion of aquaporin-4 in APP/PS1 mice exacerbates brain $A \beta$ accumulation and memory deficits. Mol Neurodegener 10: https://doi.org/10.1186/s13024-015-0056-1, 1, 58

12. Zeppenfeld DM, Simon M, Haswell JD, et al (2017) Association of perivascular localization of aquaporin-4 with cognition and Alzheimer disease in aging brains. JAMA Neurol 74:91-99. https://doi.org/10.1001/jama neurol.2016.4370, 1

13. Abe Y, Ikegawa N, Yoshida K, et al (2020) Behavioral and electrophysiological evidence for a neuroprotective role of aquaporin- 4 in the 5 xFAD transgenic mice model. Acta Neuropathol Commun 8:1-15. https://doi.org/10.1186/s404 78-020-00936-3, 1

14. Watanabe-Matsumoto S, Moriwaki Y, Okuda T, et al (2018) Dissociation of blood-brain barrier disruption and disease manifestation in an aquaporin-4deficient mouse model of amyotrophic lateral sclerosis. Neurosci Res 133: 48-57. https://doi.org/10.1016/j.neures.2017.11.001

15. Zhao ZA, Li P, Ye SY, et al (2017) Perivascular AQP4 dysregulation in the hippocampal CA1 area after traumatic brain injury is alleviated by adenosine A2A receptor inactivation. Sci Rep 7:. https://doi.org/10.1038/s41 598-017-02505-6, 1, 2254

16. Lee TS, Eid T, Mane S, et al (2004) Aquaporin-4 is increased in the sclerotic hippocampus in human temporal lobe epilepsy. Acta Neuropathol 108:493-502. https:/doi.org/10.1007/s00401-004-0910-7, 6
17. Binder DK, Oshio K, Ma T, Verkman AS, Manley GT (2004) Increased seizure threshold in mice lacking aquaporin-4 water channels. Neuroreport 15:259-262. https://doi.org/10.1097/00001756-200402090-00009, 2

18. Tradtrantip L, Jin BJ, Yao X, et al (2017) Aquaporin-targeted therapeutics: State-of-the-field. Adv Exp Med Biol 969:239-250. https://doi.org/10.1007/ 978-94-024-1057-0_16

19. Verkman AS, Anderson MO, Papadopoulos MC (2014) Aquaporins: important but elusive drug targets. Nat Rev Drug Discov 13:259-277. https://doi.org/1 $0.1038 / \mathrm{nrd} 4226,4$

20. Clément T, Rodriguez-Grande B, Badaut J (2020) Aquaporins in brain edema J Neurosci Res 98:9-18. https://doi.org/10.1002/jnr.24354, 1

21. Michinaga S, Koyama $Y$ (2015) Pathogenesis of brain edema and investigation into anti-edema drugs. Int J Mol Sci 16:9949-9975. https://doi.org/10.33 90/ijms16059949, 12

22. De Castro Ribeiro M, Hirt L, Bogousslavsky J, et al (2006) Time course of aquaporin expression after transient focal cerebral ischemia in mice. J Neurosci Res 83:1231-1240. https://doi.org/10.1002/jnr.20819, 7

23. Hirt L, Ternon B, Price M, Mastour N, Brunet JF, Badaut J (2009) Protective role of early Aquaporin 4 induction against postischemic edema formation. J Cereb Blood Flow Metab 29:423-433. https://doi.org/10.1038/jcbfm.2 $008.133,2$

24. Igarashi H, Huber VJ, Tsujita M, Nakada T (2011) Pretreatment with a novel aquaporin 4 inhibitor, TGN-020, significantly reduces ischemic cerebral edema. Neurol Sci 32:113-116. https://doi.org/10.1007/s10072-010-0431-1, 1

25. Yao X, Derugin N, Manley GT, Verkman AS (2015) Reduced brain edema and infarct volume in aquaporin-4 deficient mice after transient focal cerebral ischemia. Neurosci Lett 584:368-372. https://doi.org/10.1016/j.neulet.2014.10.040

26. Akdemir G, Ratelade J, Asavapanumas N, Verkman AS (2014) Neuroprotective effect of aquaporin-4 deficiency in a mouse model of severe global cerebral ischemia produced by transient 4-vessel occlusion. Neurosci Lett 574:70-75. https://doi.org/10.1016/j.neulet.2014.03.073

27. Manley GT, Binder DK, Papadopoulos MC, Verkman AS (2004) New insights into water transport and edema in the central nervous system from phenotype analysis of aquaporin-4 null mice. Neuroscience 129:981-989. https://doi.org/10.1016/j.neuroscience.2004.06.088, 4

28. Shi WZ, Qi LL, Fang SH, Lu YB, Zhang WP, Wei EQ (2012) Aggravated chronic brain injury after focal cerebral ischemia in aquaporin-4-deficient mice. Neurosci Lett 520:121-125. https://doi.org/10.1016/j.neulet.2012.05.052, 1

29. Shi WZ, Zhao CZ, Zhao B, et al (2012) Aggravated inflammation and increased expression of cysteinyl leukotriene receptors in the brain after focal cerebral ischemia in AQP4- deficient mice. Neurosci Bull 28:680-692. https://doi.org/10.1007/s12264-012-1281-z, 6

30. Le Bihan D (2007) The "wet mind": water and functional neuroimaging. Phys Med Biol 52:R57-R90. https://doi.org/10.1088/0031-9155/52/7/R02, 7

31. Assaf $Y$, Ben-Bashat D, Chapman J, et al (2002) High b-value q-space analyzed diffusion-weighted MRI: Application to multiple sclerosis. Magn Reson Med 47:115-126. https://doi.org/10.1002/mrm.10040, 1

32. Le Bihan D, Breton E, Lallemand D, et al (1988) Separation of diffusion and perfusion in intravoxel incoherent motion MR imaging. Radiology 168:497-505. https://doi.org/10.1148/radiology.168.2.3393671, 2

33. Clark CA, Le Bihan D (2000) Water diffusion compartmentation and anisotropy at high b values in the human brain. Magn Reson Med 44:852-859. https://doi.org/10.1002/1522-2594(200012)44:6<852::AID-MRM5>3.0.CO;2-A, 6

34. Obata T, Kershaw J, Tachibana Y, et al (2018) Comparison of diffusion weighted MRI and anti-Stokes Raman scattering (CARS) measurements of the inter-compartmental exchange-time of water in expression-controlled aquaporin 4 cells. Sci Rep 8:. https://doi.org/10.1038/s41598-018-36264-935.

35. Andrasko J (1976) Water diffusion permeability of human erythrocytes studied by a pulsed gradient NMR technique. BBA Gen Subj 428:304-311. https://doi.org/10.1016/0304-4165(76)90038-6, 2

36. Kärger J, Pfeifer H, Heink W (1988) Principles and application of self-diffusion measurements by nuclear magnetic resonance. Advances in Magnetic and Optical Resonance. Vol 12. p 1-89. https://doi.org/10.1016/B978-0-12-02 5512-2.50004-X.

37. Lee JH, Springer CS (2003) Effects of equilibrium exchange on diffusionweighted NMR signals: The diffusigraphic "shutter-speed." Magn Reson Med 49:450-458. https://doi.org/10.1002/mrm.10402, 3

38. Lätt J, Nilsson M, van Western D, et al (2009) Diffusion-weighted MRI measurements on stroke patients reveal water-exchange mechanisms in sub-acute ischaemic lesions. NMR Biomed 22:619-628. https://doi.org/10.1 002/nbm.1376, 6 
39. Li H, Jiang X, Xie J, McIntyre JO, Gore JC, Xu J (2016) Time-dependent influence of cell membrane permeability on MR diffusion measurements. Magn Reson Med 75:1927-1934. https://doi.org/10.1002/mrm.25724, 5

40. Ibata K, Takimoto S, Morisaku T, Miyawaki A, Yasui M (2011) Analysis of aquaporin-mediated diffusional water permeability by coherent anti-Stokes Raman scattering microscopy. Biophys J 101:2277-2283. https://doi.org/10.1 016/j.bpj.2011.08.045, 9

41. Kato J, Takai Y, Hayashi MK, et al (2014) Expression and localization of aquaporin-4 in sensory ganglia. Biochem Biophys Res Commun 451:562-567. https://doi.org/10.1016/j.bbrc.2014.08.026, 4

42. Ikeshima-Kataoka H, Abe Y, Abe T, Yasui M (2013) Immunological function of aquaporin-4 in stab-wounded mouse brain in concert with a proinflammatory cytokine inducer, osteopontin. Mol Cell Neurosci 56:65-75. https://doi.org/10.1016/j.mcn.2013.02.002

43. Tamura A, Graham DI, McCulloch J, Teasdale GM (1981) Focal cerebral ischaemia in the rat: I. Description of technique and early neuropathological consequences following middle cerebral artery occlusion. J Cereb Blood Flow Metab 1:53-60. https://doi.org/10.1038/jcbfm.1981.6, 1

44. Brint S, Jacewicz M, Kiessling M, Tanabe J, Pulsinelli W (1988) Focal brain ischemia in the rat: Methods for reproducible neocortical infarction using tandem occlusion of the distal middle cerebral and ipsilateral common carotid arteries. J Cereb Blood Flow Metab 8:474-485. https://doi.org/10.103 8/jcbfm.1988.88, 4

45. Garcia JH, Yoshida Y, Chen H et al (1993) Progression from ischemic injury to infarct following middle cerebral artery occlusion in the rat. Am J Pathol 142:623-635

46. McCullough LD, Liu F (2011) Middle cerebral artery occlusion model in rodents: Methods and potential pitfalls. J Biomed Biotechnol 2011:. https://doi.org/10.1155/2011/464701, 2011, 1, 9

47. Krueger M, Mages B, Hobusch C, Michalski D (2019) Endothelial edema precedes blood-brain barrier breakdown in early time points after experimental focal cerebral ischemia. Acta Neuropathol Commun 7:17. https://doi.org/10.1186/s40478-019-0671-0, 1

48. Ogura A, Miyai A, Maeda F, et al (2003) [Accuracy of signal-to-noise ratio measurement method for magnetic resonance images]. Nihon Hoshasen Gijutsu Gakkai Zasshi 59:508-513. https://doi.org/10.6009/jjrt.kj00003174111, 4

49. Debaker C, Djemai B, Ciobanu L, Tsurugizawa T, le Bihan D (2020) Diffusion $M R I$ reveals in vivo and non-invasively changes in astrocyte function induced by an aquaporin-4 inhibitor. PLoS One 15:. https://doi.org/10.1371/ journal.pone.0229702, 15, 5, e0229702

50. Yao X, Hrabětová S, Nicholson C, Manley GT (2008) Aquaporin-4-deficient mice have increased extracellular space without tortuosity change. J Neurosci 28:5460-5464. https://doi.org/10.1523/JNEUROSCI.0257-08.2008, 21

51. Nicchia GP, Frigeri A, Liuzzi GM, Svelto M (2003) Inhibition of AQP4 expression in astrocytes by RNAi determines alterations in cell morphology, growth, and water transport and induces changes in ischemia related genes. FASEB J 17:. https://doi.org/10.1096/fj.02-1183fje, 17, 11, 1, 21

52. Risher WC, Andrew RD, Kirov SA (2009) Real-time passive volume responses of astrocytes to acute osmotic and ischemic stress in cortical slices and in vivo revealed by two-photon microscopy. Glia 57:207-221. https://doi. org/10.1002/glia.20747, 2

53. Badaut J, Ashwal S, Obenaus A (2011) Aquaporins in cerebrovascular disease: a target for treatment of brain edema? Cerebrovasc Dis 31:521-531. https://doi.org/10.1159/000324328, 6

54. Le Bihan D, Breton E, Lallemand D, et al (1986) MR imaging of intravoxe incoherent motions: Application to diffusion and perfusion in neurologic disorders. Radiology 161:401-407. https://doi.org/10.1148/radiology.161.2.3 763909, 2

55. Urushihata T, Takuwa $H$, Seki C, et al (2018) Water diffusion in the brain of chronic hypoperfusion model mice: a study considering the effect of blood flow. Magn Reson Med Sci 17:318-324. https://doi.org/10.2463/mrms.mp.2 017-0149, 4

56. Nicholson C, Hrabětová S (2017) Brain extracellular space: the final frontier of neuroscience. Biophys J 113:2133-2142. https://doi.org/10.1016/j.bpj.2017. 06.052, 10

57. Horner A, Pohl P (2018) Single-file transport of water through membrane channels. Faraday Discuss 209:9-33. https://doi.org/10.1039/c8fd00122g, 0

58. Mitsui T, Rose MK, Fomin E, Ogletree DF, Salmeron M (2002) Water diffusion and clustering on Pd(111). Science (80- ) 297:1850-1852. https://doi.org/1 $0.1126 /$ science.1075095, 297, 5588, 1850, 1852
59. Zeuthen T (2001) How water molecules pass through aquaporins. Trends Biochem Sci 26:77-79. https://doi.org/10.1016/S0968-0004(00)01778-3, 2

60. Tachibana A, Tachibana Y, Kershaw J, Sano H, Fukushi M, Obata T (2018) Comparison of glass capillary plates and polyethylene fiber bundles as phantoms to assess the quality of diffusion tensor imaging. Magn Reson Med Sci 17:251-258. https://doi.org/10.2463/mrms.mp.2017-0079, 3

61. Németh-Cahalan KL, Hall JE (2000) pH and calcium regulate the water permeability of aquaporin 0. J Biol Chem 275:6777-6782. https://doi.org/1 0.1074/jbc.275.10.6777, 10

62. Németh-Cahalan KL, Kalman K, Hall JE (2004) Molecular basis of pH and Ca2+ regulation of aquaporin water permeability. J Gen Physiol 123:573-580. https:/doi.org/10.1085/jgp.200308990, 5

63. Mutch WAC, Hansen AJ (1984) Extracellular pH changes during spreading depression and cerebral ischemia: Mechanisms of brain $\mathrm{pH}$ regulation. J Cereb Blood Flow Metab 4:12-27. https://doi.org/10.1038/jcbfm.1984.3,

64. Beppu K, Sasaki T, Tanaka KF, et al (2014) Optogenetic countering of glial acidosis suppresses glial glutamate release and ischemic brain damage. Neuron 81:314-320. https://doi.org/10.1016/j.neuron.2013.11.011, 2

65. Lampinen B, Lätt J, Wasselius J, Westen D, Nilsson M (2021) Time dependence in diffusion MRI predicts tissue outcome in ischemic stroke patients. Magn Reson Med 86:754-764. https://doi.org/10.1002/mrm.28743, 2

66. Lee HH, Papaioannou A, Novikov DS, Fieremans E (2020) In vivo observation and biophysical interpretation of time-dependent diffusion in human cortical gray matter. Neuroimage 222:. https://doi.org/10.1016/j.neuroima ge.2020.117054, 117054

\section{Publisher's Note}

Springer Nature remains neutral with regard to jurisdictional claims in published maps and institutional affiliations.

\section{Submit your manuscript to a SpringerOpen ${ }^{\circ}$ journal and benefit from:}

- Convenient online submission

- Rigorous peer review

- Open access: articles freely available online

High visibility within the field

- Retaining the copyright to your article

Submit your next manuscript at $>$ springeropen.com 